\title{
Mechanical Modeling of Initiation of Localized Yielding Under Plane Stress Conditions in Rigid-Rigid Polymer Alloys
}

\author{
H. J. SUE,* R. A. PEARSON, ** and A. F. YEE*** \\ Macromolecular Research Center and \\ Department of Materials Science and Engineering \\ University of Michigan, Ann Arbor, Michigan 48109
}

\begin{abstract}
Two-dimensional Finite Element Method simulations, which involve consideration of the nonlinearity of a material, have been conducted to gain understanding about the rigid-rigid polymer toughening concept we proposed. The simulation results for the plane stress condition indicate that as long as the inclusion phase possesses i) a $60 \%$ difference in the tangent modulus from that of the matrix at any given strain level prior to failure or ii) smaller yield or craze stain than the yield strain of the matrix, then, localized shear yielding will occur around the inclusion. A toughened rigid-rigid polymer alloy system can then be obtained. The plain strain case is also discussed with an implementation of the rigid-rigid polymer toughening concept.
\end{abstract}

\section{INTRODUCTION}

$\mathrm{T}$ he beneficial combination of a wider range of applications, higher production rates, and improved mechanical and physical properties has led to the development of a new generation of engineering polymers, i.e., rigid-rigid" polymer alloys. Acrylonitrile-butadiene-styrene/polycarbonate (ABS/PC), $\dagger$ polyamide $6,6 /$ polyphenylene oxide (PA/PPO), PC/ABS, polyamide 6/ABS, and polybutylene terephthalate/polycarbonate $(\mathrm{PBT} / \mathrm{PC})$ are typical examples of these alloys. All of these alloys are commercially available. Among them, for example, the PA/PPO alloy possesses combinations of good solvent resistance, high strength and high modulus, low creep and post-mold shrinkage, wide processing window, outstanding dimensional stability at high temperature $\left(200^{\circ} \mathrm{C}\right)$, and high impact strength at low temperatures $\left(-40^{\circ} \mathrm{C}\right)$. These properties cannot be obtained from the individual PA or PPO homopolymers alone. However, the process by which polymer pairs are selected for maximum toughness, as well as good yield strength, modulus, and creep resistance, remains an enigma.

The recent introduction of the rigid-rigid polymer toughening concept $(1-5)$ has provided new ground

*Present address: Dow Chemical USA, B-1470, Texas Polymer Center. Freeport, TX 77541.

* Present address: Department of Materials Science and Engineering, Lehigh Unlversity, Bethlehem, PA 18015

***To whom correspondence should be addressed.

${ }^{9}$ Rigid means non-elastomeric in the content of this work.

†Throughout the context of this work the first Hsted polymer, 1.e., ABS

in this case, indicates the matrix phase: the second listed polymer. i.e.

$\mathrm{PC}$, indicates the inclusion phase. for structural applications of polymeric materials (1-19). This rigid-rigid polymer toughening concept is, in fact, derived from the rubber toughening concept we previously proposed (20). In short, the rigid-rigid polymer toughening concept suggests that as long as the second-phase rigid polymer can help i) relieve the triaxial tension in front of the crack tip and ii) generate numerous stress concentration sites around the crack tip sequentially, like in the case of rubber particles in the rubber toughened epoxy systems, then, a rigid polymer matrix can be toughened by the introduction of the second-phase rigid polymer.

Research on the micromechanics of two-phase metal and ceramic alloys has been extensively conducted in the last two decades (21-35). Yet, owing to the dissimilar deformation mechanisms between polymers and ceramics as well as metal materials, the application of metal and ceramic alloy toughening concepts directly to polymer alloy systems still awaits verification. In the field of polymers, research has been concentrated in either one-phase yielding behavior of polymers, or plastic deformations of polymers due to the presence of the inclusion phase, where the latter only deforms elastically $(36-49)$. In such cases, the rigid-rigid polymer toughening concept cannot be fully explored, i.e., the effect of stress concentration due to the anelastic and plastic deformations of the inclusion phase on the matrix phase is not realized. Thus, to examine the feasibility of the rigid-rigid polymer toughening concept, it is necessary that one studies the micromechanics of polymer yielding due to anelastic and plastic deformations of inclusions. 
As mentioned earlier, there are many rigid-rigid polymer alloys available in the marketplace. These commercially available rigid-rigid polymer alloys were, however, made by trial and errors. No criteria or guidelines have been used in making these increasingly important polymer alloys. Computer simulations using the Finite Element Method (50) (FEM) is needed to predict the stress concentration effect due to the dispersed phase, given the nonlinear mechanical nature of polymers. There are several papers that have attempted to correlate results of computer simulations using the linear elastic assumptions with experimental results (45-49) on the yielding behavior of polymers. These correlations may not only be inappropriate but could also be very misleading. Moreover, as pointed out earlier, there has never been any attempt to study the $\sigma-\epsilon$ field of a polymer alloy system when the inclusion phase undergoes anelastic and plastic deformations. It is, therefore, worthwhile to verify these interesting aspects in the nonlinear case. The current research is meant to gain insights about the feasibility of the rigid-rigid polymer toughening concept and look into the practicality of FEM simulations on the nonlinear behaviors of polymers.

We have previously studied the deformation behavior of a polycarbonate plate with a circular hole both experimentally and numerically, using 2-D FEM nonlinear material property simulations (51). The 2-D FEM results correlate extremely well with the experimental observations. Given the confidence obtained from the previous work, various combinations of polymer pairs were studied using 2-D FEM in the present paper. These rigid-rigid polymer pairs are listed in Table 1. The 2-D FEM simulation results do provide useful insights for the selection of toughened rigid-rigid polymer pairs. In addition, in cases where the plane strain condition prevails, the rigid-rigid polymer toughening concept is incorporated for the selection of toughened rigidrigid polymer pairs.

\section{MODELING USING 2-D FEM}

The detail of the 2-D FEM modeling can be found from the previous paper (51). Only a brief description of the modeling procedure is given here.

The 2-D FEM simulation is carried out using the MSC/NASTRAN FEM package (64A, 1985 version).
Many assumptions have been made in this work:

i) isotropy of the matrix and the inclusion phases; ii) cylindrical dispersed phase with the cylinder axis perpendicular to the plane of the model; iii) perfect. adhesion between the phases; iv) no interaction between dispersed inclusions; v) negligible thermal stress at the interface; vi) piecewise linear stressstrain curve (Fig. 1); vii) time independent deformation.Furthermore, the von Mises yielding criterion is used to determine the onset of the yielding of polymers.

The FEM model is subjected to a far field tensile stress. In the third (thickness) direction, a thin ( $\sigma_{3}=$ 0) plate is assumed (See Fig. 2), i.e., under plane stress condition. A rather simple but effective FEM mesh was generated using the PATRAN package (Fig. 3). It is found that this simplified FEM mesh is sufficient for the present purpose. The deformation of many rigid-rigid polymer pairs were simulated in these computer experiments (see Table 1).

\section{RESULTS}

In this study, owing to the nature of the 2-D FEM simulations, the results are only relevant for the plane stress condition. The FEM results cannot be used to relate to the material behavior in front of a sharp crack tip, where the plane strain condition prevails. The rigid-rigid polymer toughening concept, as pointed out earlier, has to be incorporated to modify the FEM results.

In order to determine what criteria should be used for obtaining a toughened rigid-rigid polymer alloy,

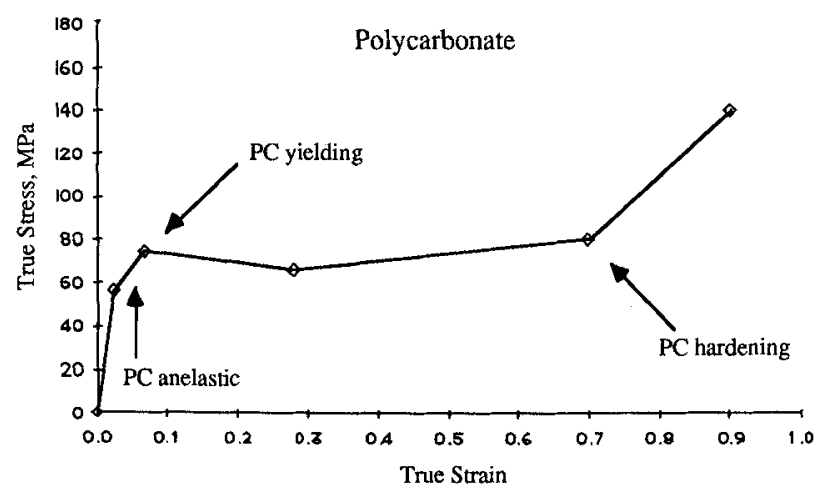

Fig. 1. Typical piecewise linear stress-strain curve used for FEM simulations.

Table 1. List of Polymer Pairs Being Computer Simulated Using 2-D FEM. Note that the numbers in this table are only intended for showing the qualitative trend of the alloy systems studied.

\begin{tabular}{lcccc}
\hline & $\begin{array}{c}\text { Elastic Modulus, MPa } \\
\text { (Matrix/Inclusion) }\end{array}$ & $\begin{array}{c}\text { Poisson's Ratio, } \\
\text { (Matrix/Inclusion) }\end{array}$ & $\begin{array}{c}\text { Yield (Craze) Strain } \\
\text { (Matrix/Inclusion) }\end{array}$ & $\begin{array}{c}\text { Yield (Craze) Stress, MPa } \\
\text { (Matrix/Inclusion) }\end{array}$ \\
\hline PC/glass & $2400 / 80000(=0.03)$ & $0.42 / 0.22$ & $0.07 /-$ & $75 /-$ \\
PA/PC & $680 / 2400(=0.28)$ & $0.45 / 0.42$ & $0.2 / 0.07$ & $55 / 75$ \\
PC/PS & $2400 / 3400(=0.72)$ & $0.42 / 0.38$ & $0.07 / 0.02$ & $75 / 40$ \\
PEl/PS & $3400 / 3400(=1.00)$ & $0.40 / 0.38$ & $0.11 / 0.02$ & $137 / 40$ \\
PEI/PC & $3400 / 2400(=1.39)$ & $0.40 / 0.42$ & $0.11 / 0.07$ & $137 / 75$ \\
PC/PA & $2400 / 680(=3.53)$ & $0.42 / 0.45$ & $0.07 / 0.2$ & $75 / 55$ \\
PC/PE & $2400 / 190(=12.6)$ & $0.42 / 0.47$ & $0.07 / 0.25$ & $75 / 8$ \\
PC/rubber & $2400 / 8(=300)$ & $0.42 / 0.5$ & $0.07 /-$ & $75 /-$ \\
PC/hole & $2400 /-(=\infty)$ & $0.42 /-$ & $0.07 /-$ & $75 /-$ \\
\hline
\end{tabular}




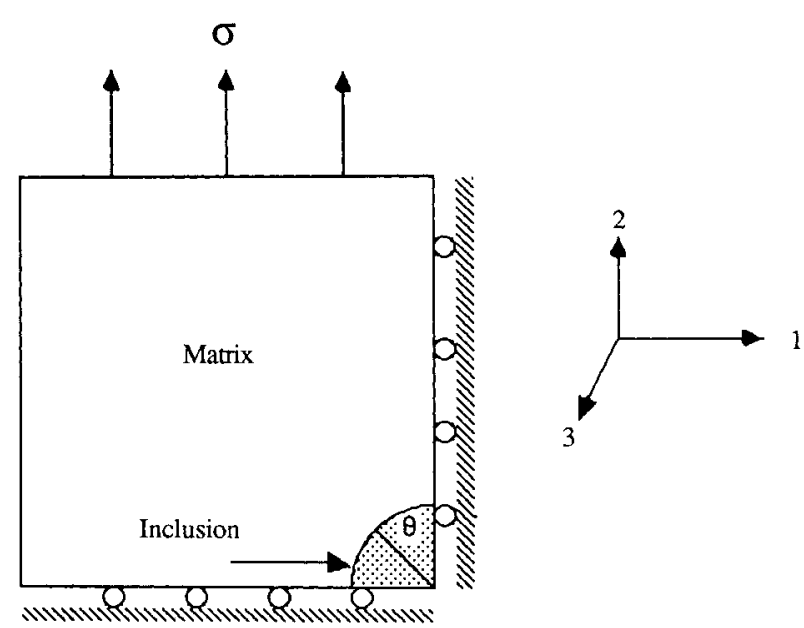

Fig. 2. Schematic drawing of the FEM model; $\theta$ is defined as the angle between the tensile direction and the direction from the center of the inclusion to the location of the maximum $\tau_{\text {oct }}$.

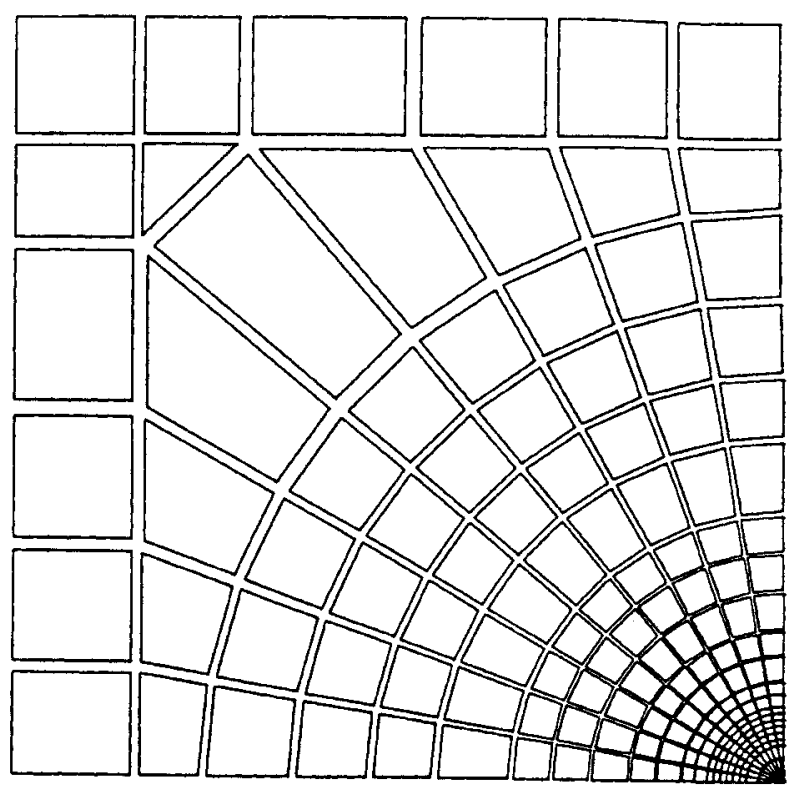

Fig. 3. FEM model mesh generated using the PATRAN preprocessor. This simplified mesh is sufficient for the present work.

six model rigid-rigid polymer pairs, along with $\mathrm{PC} /$ glass, $\mathrm{PC} /$ hole, and $\mathrm{PC} /$ rubber (Table 1) are simulated using 2-D FEM. The PC/glass, PC/hole, and $\mathrm{PC} /$ rubber systems were studied to check the validity of the 2-D FEM simulations. The simulation results on these three systems agree well with the experimental observations conducted by others $(43-45,52)$ on the locations where the shear bands initiate and grow along the interface. These good correlations along with the $\mathrm{PC} /$ hole experiments (51) provide confidence in the 2-D FEM simulated results of the selected rigid-rigid polymer alloys (See Table 1) to be presented below.

Among all the rigid-rigid polymer pairs being studied, it is found that two particular systems, where the matrix and the inclusion phases possess unique combinations of $\sigma-\epsilon$ behaviors, are highly significant. These are: 1) poly(ether imide)/polycarbonate (PEI/PC) and 2) poly(ether imide)/polystyrene (PEI/PS). The above two systems become effective only when the yielding or crazing stresses between the matrix and the inclusion are comparable, i.e., within a factor of three (see the discussion section below). Under such conditions, these two kinds of rigid-rigid polymer pairs are found to be effective in triggering localized shear banding under the plane stress condition.

\section{The PEI/PC System}

In this model, a PC inclusion is embedded into a PEI matrix and loaded under tension. The PEI/PC system typifies a system where the inclusion phase, i.e., PC, possesses a more pronounced anelastic response as well as a smaller yield strain than those of the matrix. The $\sigma-\epsilon$ curves of these two polymers, idealized from experimental true stress true strain behavior as piecewise continuous curves, are shown in Fig. 4. These curves show that the two polymers have similar elastic moduli $(\approx 40 \%$ difference) but PEI possesses a rather high yield stress value. When the system is tested under tension, as shown in Fig. 5 , the anelastic and plastic deformations of the PC inclusion do alter the octahedral shear stress $\left(\tau_{o c t}\right)$ concentration of the matrix around the inclusion at various strain levels. In the linear elastic regime, the stress concentration build-up is too small to cause localized yielding in the PEI matrix, i.e., the stress concentration is low before the anelastic deformation and yielding of the PC inclusion takes place. Upon the anelastic deformation of $\mathrm{PC}$, the stress concentration in the PEI matrix begins to build up. If the stress concentration factor multiplied by the applied far field stress is higher than the yield stress of PEI, then, the localized matrix shear yielding will occur at the PEI/PC interface. On the other hand, if the yield stress of the matrix is still too high, no plastic deformation will take place. However, when the PC inclusion begins to shear yield upon further

PEI/PC

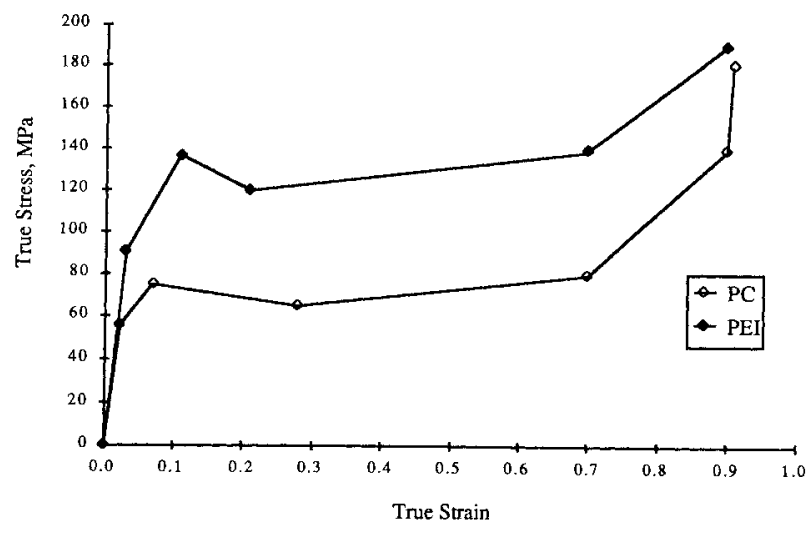

Fig. 4. True stress-strain curve of poly(ether imide) and polycarbonate. 
loading, an abrupt increase of stress concentration factor in the PEI matrix toward that of the rubber particle (ca. three) (4) can be reached. This is because the tangent (or instantaneous) modulus of the yielding $\mathrm{PC}$ inclusion is approaching zero. At this point, the PEI matrix begins to undergo localized shear yielding in the vicinity of the PC particle, i.e., the true tensile yield stress* of PC (75 MPa) (53) multiplied by the stress concentration factor (i.e., three) is greater than the tensile yield stress of PEI (ca. $139 \mathrm{MPa}$ ). When the localized yielding of PEI takes place, a significant increase in toughness of the system may ensue.

The constant $\tau_{\text {oct }}$ contours obtained using the 2-D FEM simulation process are given in Fig. 6. Figure 6 shows the constant $\tau_{\text {oct }}$ contour plots of PEI/PC at various applied tensile stages. In the elastic regime, the maximum $\tau_{o c t}$ is located at the equatorial region and the $\tau_{o c t}$ stress concentration is ca. 1.2. When the PC inclusion begins to undergo anelastic deformation, the $\tau_{\text {oct }}$ rises to about 1.3. When the shear yielding of PC inclusion occurs, the $\tau_{\text {oct }}$ stress concentration increases abruptly. ${ }^{\dagger}$ As soon as the PEI matrix begins to shear yield, the location of the maximum $\tau_{\text {oct }}$ shifts upward from the equatorial region, similar to the case demonstrated in the $\mathrm{PC} /$ hole system (51).

On the other hand, if the inclusion phase has a similar anelastic deformation but with a larger yield strain than the matrix, as in the case of the PC (matrix) and PA (inclusion) system, the inclusion

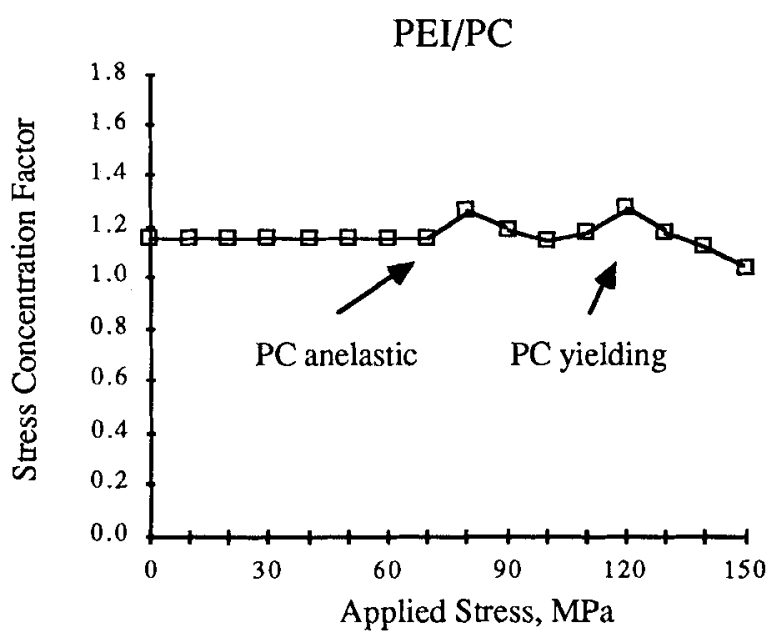

Fig. 5. Octahedral stress concentration factor v.s. applied stress for $\mathrm{PEI} / \mathrm{PC}$ system. In the linear range, the stress conc. is about 1.2. As PC begins to undergo anelastic deformation and yielding, the stress concentration rises and has a max. value at the point that PC starts to yield. It is believed that at the time PC yields, it behaves like a rubber particle. Note that no cavitation and debonding are assumed.

*Under uniaxial tension, the octahedral shear stress equals to the applied stress multiplied by 0.471 .

The reason why the stress concentration does not rise to ca. 3 is because the load increment used in the nonlinear simulation process is too coarse to produce the stress concentration value obtained from an analytical solution. may not be able to shear yield until the yielding of the matrix occurs. This is because the deformation of the inclusion is restricted by the surrounding matrix. The inclusion cannot shear yield until the

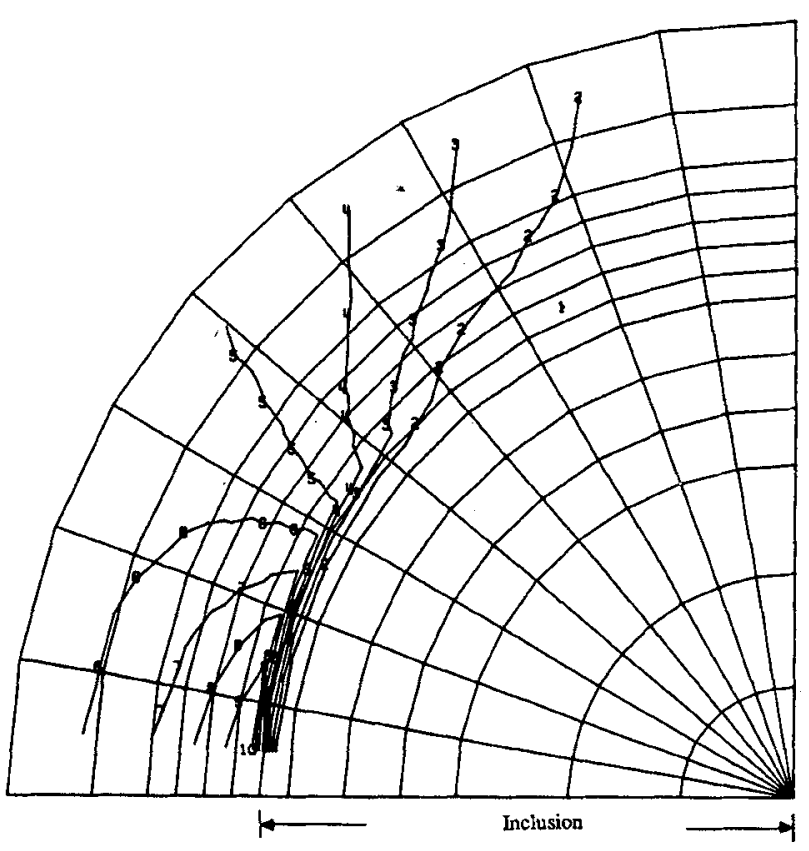

(a)

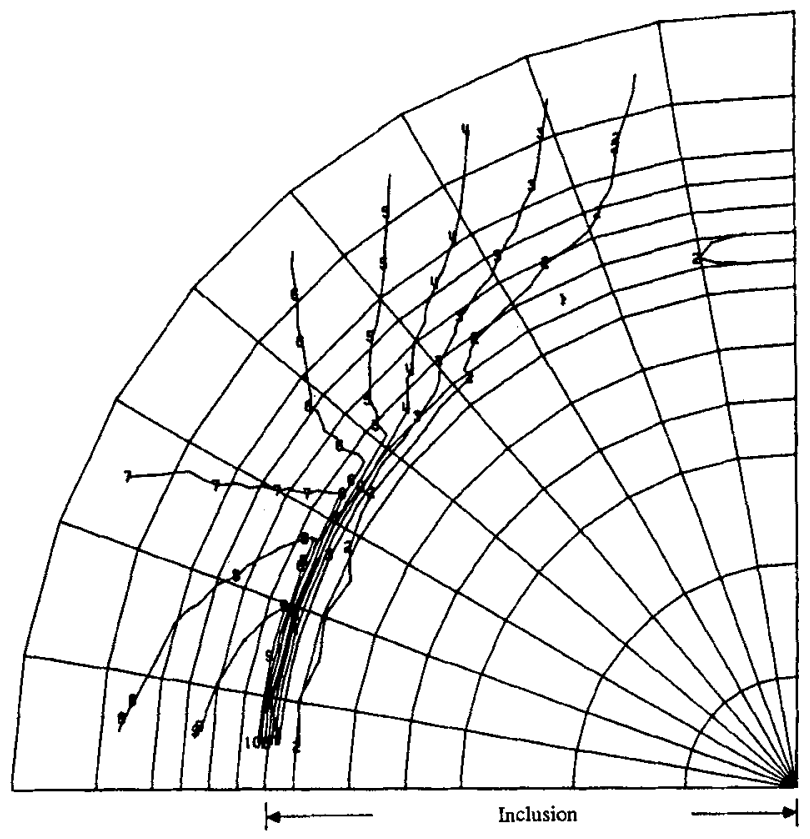

(b)

Fig. 6. The $\tau_{\text {oct }}$ contour plots of the PEI/PC system. The maximum $\tau_{\text {oct }}$ (curve number 10 ) is located at the equatorial region when the stress state is still in the linear elastic regime, as shown in (a); when the material has a local stress state which exceeds the yield stress, the maximum $\tau_{\text {oct }}$ concentration begins to intensify, as shown in (b); if the load is further increased, the $\tau_{\text {oct }}$ shift upward like in the case of the PC/hole system, as shown in (c). Note that only part of the mesh is shown. 


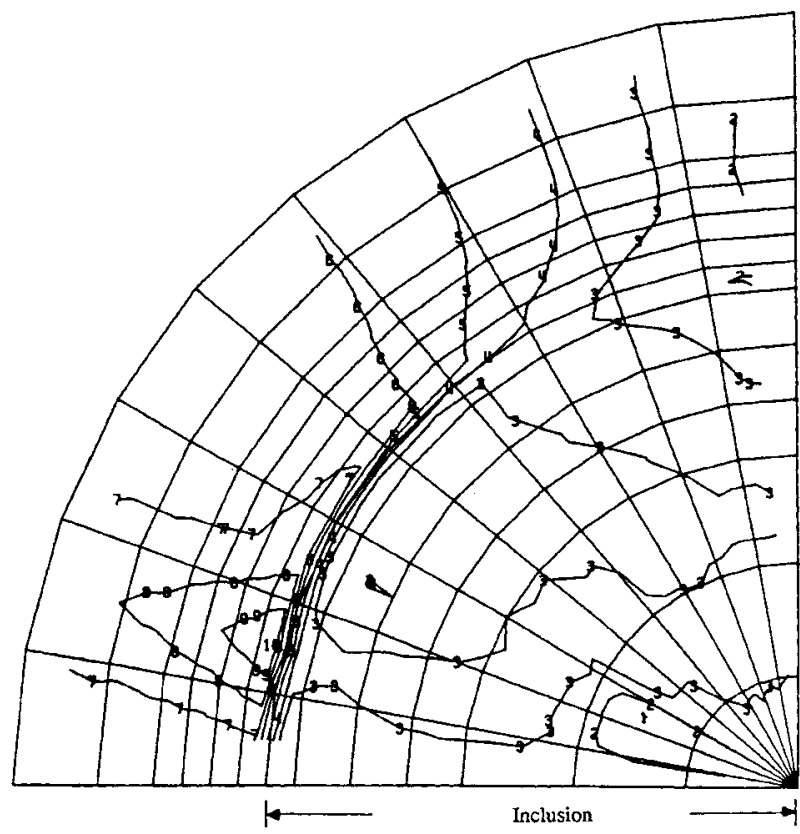

(c)

Fig. 6-Continued.

matrix undergoes large deformation. Under such a condition, the inclusion phase acts like a rigid particle and may cause brittle failure rather than widespread localized yielding of the matrix. As a result, the toughening effect would be expected to be lirnited.

\section{The PEI/PS System}

The second case for effective toughening is one where the inclusion possesses a smaller crazing strain than the yielding strain of the matrix, e.g., the $\mathrm{PEI} / \mathrm{PS}$ system. The piecewise linear $\sigma-\epsilon$ curves of PEI and PS are shown in Fig. 7. PEI and PS have approximately the same elastic constants but the inclusion PS phase has a smaller crazing strain than the yielding strain of the PEI matrix under uniaxial tension. The $\tau_{\text {oct }}$ concentration due to the formation

\section{PEI/PS}

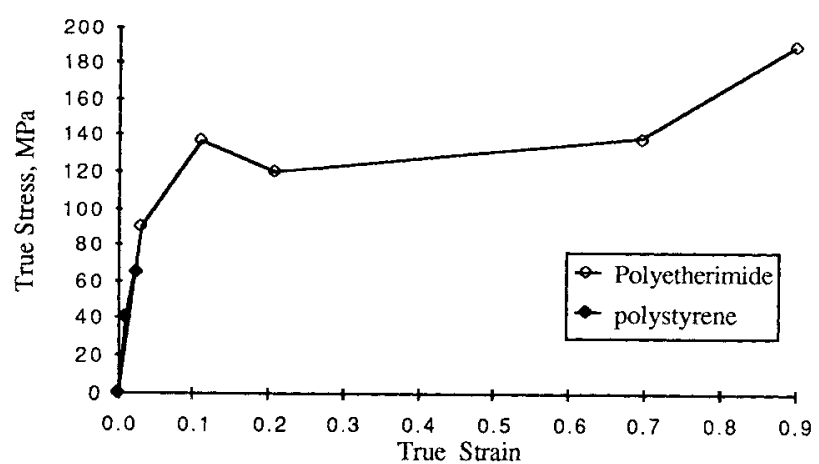

Fig. 7. True stress-strain curve of poly(ether imide) and polystyrene. of crazes in PS in the PEI matrix system is shown in Fig. 8. When the alloy is subjected to tension, the stress concentration factor is initially $\approx 1$. As the externally applied stress reaches the crazing stress of the PS inclusion, the PS begins to undergo crazing and subsequently fractures. Hence, the stress concentration factor around the interface changes from $\approx 1$ and approached $\approx 3$, when the PS inclusion can no longer bear any load $(4,54)$. Therefore, the crazing of PS causes a localized stress concentration and may cause the localized shear yielding of the PEI matrix, if the crazing process does not trigger the cracking of the matrix at the interface. The toughness of the system may therefore be greatly increased.

The $\tau_{o c t}$ contours of PEI/PS during the simulation process are given in Fig. 9. In Fig. 9, various contour plots were obtained at corresponding stages of applied tensile stress. In the elastic regime, the maximum $\tau_{o c t}$ is located approximately at the $45^{\circ}$ region along the interface and the $\tau_{\text {oct }}$ stress concentration factor here is $\approx 1$. When the PS inclusion begins to undergo crazing, the $\tau_{\text {oct }}$ stress concentration increases abruptly. Also, the location of maximum $\tau_{\text {oct }}$ shifts to the equatorial region. The instant shift of the $\tau_{\text {oct }}$ to the equatorial region is a result of the fact that the PS inclusion becomes more compliant than the PEI matrix.

The crazing strain for PS microspheres inside the PEI matrix may be far greater than the crazing strain measured in the bulk sample because of the lower probability of finding flaws in the PS microsphere. Furthermore, it is not known whether the crazes formed in the PS inclusion are effective in triggering the surrounding matrix to shear yield. By letting the crazing strain of PS microspheres be smaller than the yield strain of the surrounding PEI, and by assuming that the formation of crazes inside the PS microspheres does relieve the triaxial tension

\section{PEI/PS}

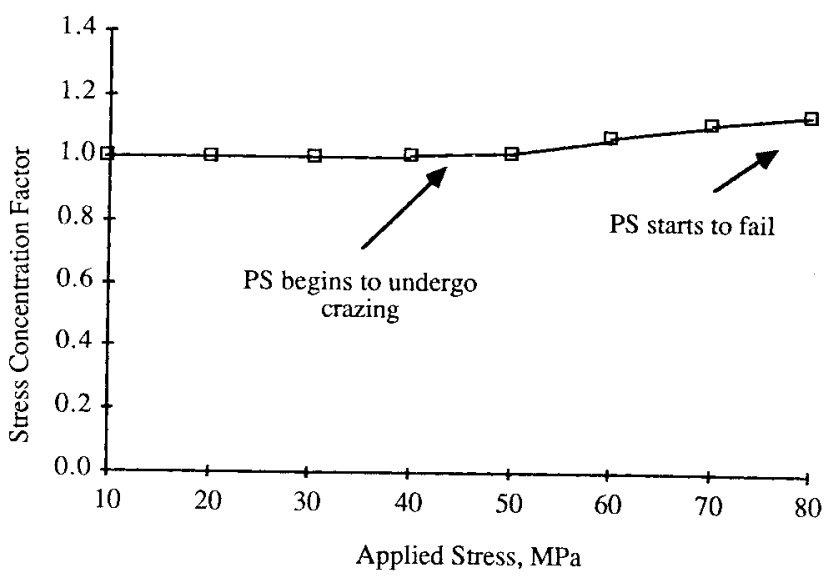

Fig. 8. Octahedral stress concentration factor v.s. applied stress for PEI/PS system. In the linear range, the stress concentration is about one. As PS begins to undergo crazing, the stress concentration rises until the point that the PS can no longer bear the load. 


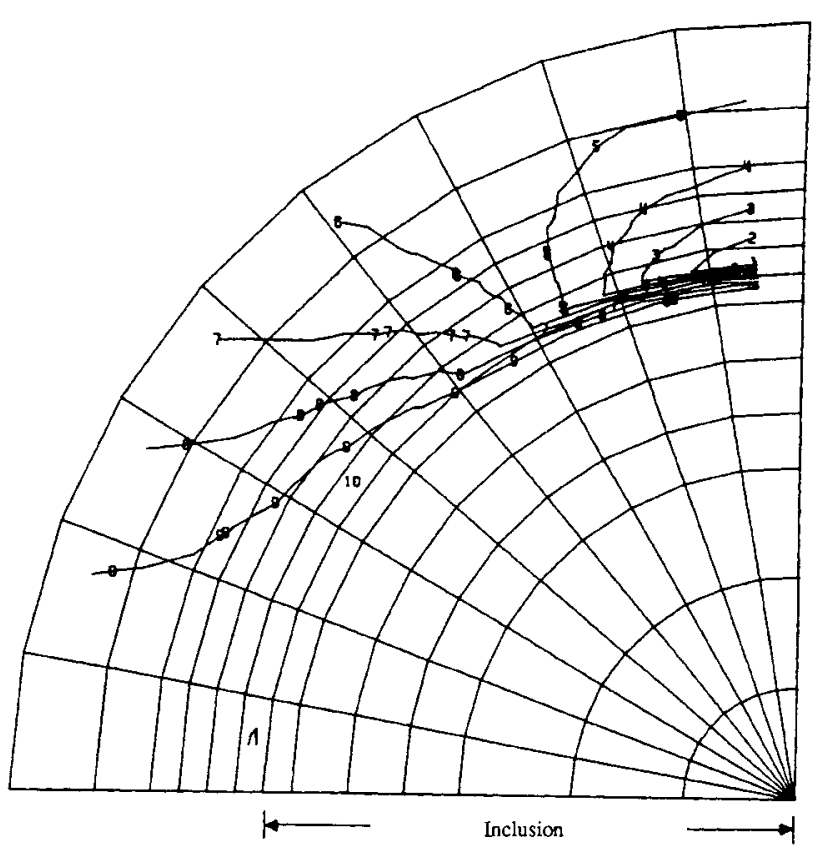

(a)

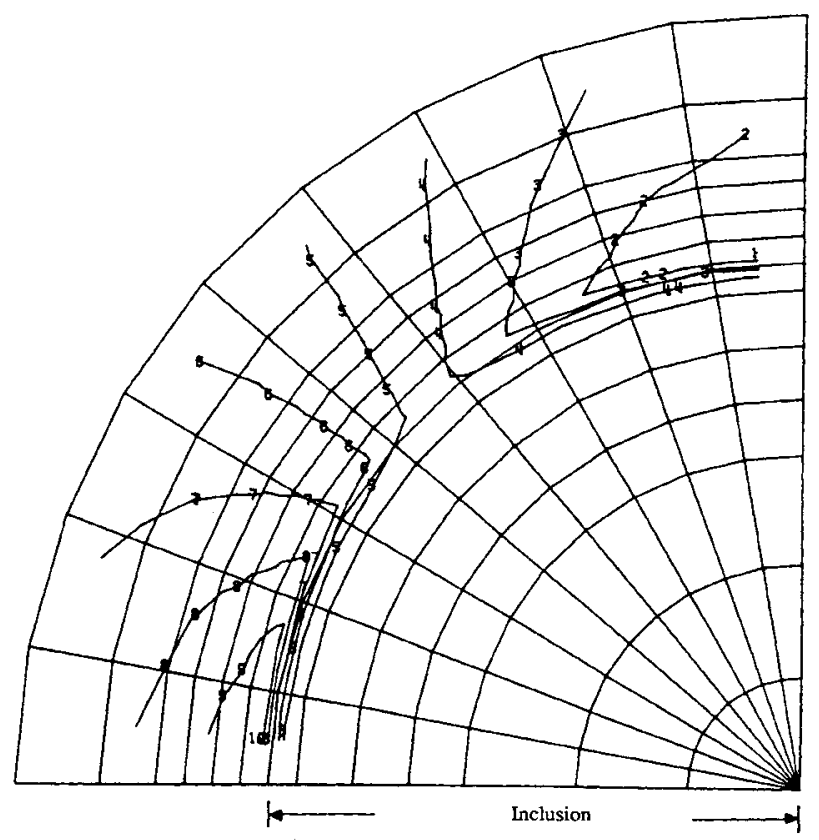

(b)

Fig. 9. The $\tau_{\text {oct }}$ contour plots of the PEI/PS system. The maximum $\tau_{\text {oct }}$ (curve number 10) is located approximately at the $45^{\circ}$ region when the stress state is still in the linear elastic regime, as shown in $(a)$; when the PS inclusion has a local stress state which exceeds the crazing stress, the PS inclusion becomes more compliant than the PEI matrix and causes the maximum $\tau_{\text {oct }}$ shifts to the equatorial region, as shown in (b); if the load is further increased, the $\tau_{\text {oct }}$ concentration intensifies and eventually reaches to that of a hole case when the PS inclusion can no longer bear the load, as shown in (c). Note that only part of the mesh is shown.

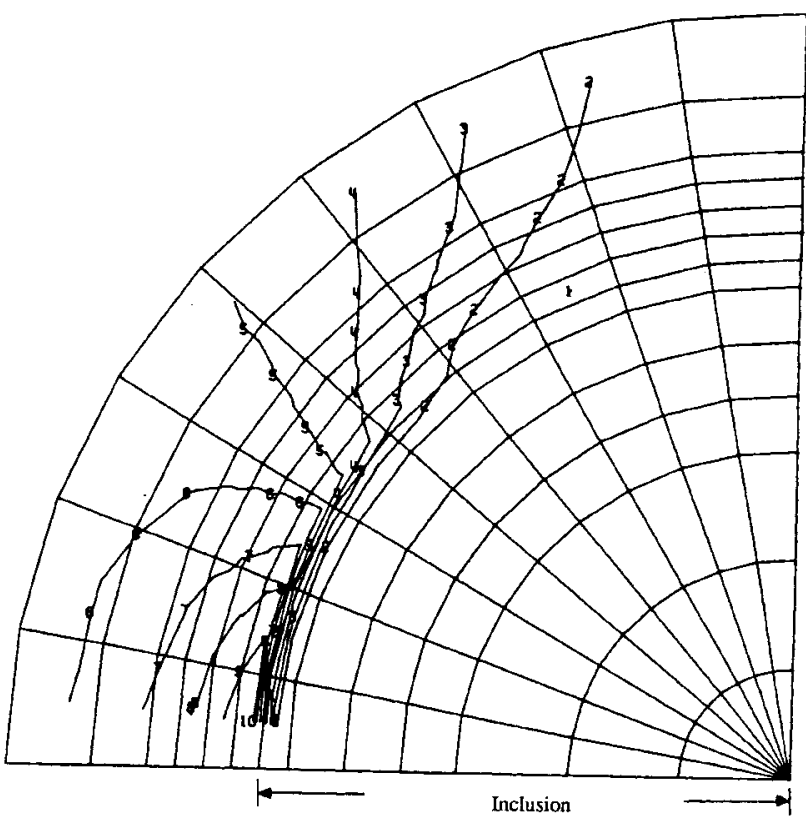

(c)

Fig. 9-Continued.

(in the case of plane strain condition), and therefore, can activate localized shear bands in the matrix, then, this type of polymer pair may be ideal for attaining the rigid-rigid polymer toughening, since, effectively, the PS does not create any stress concentration until crazing of the PS inclusion takes place. Thus, all the mechanical properties of the matrix polymer can be retained while benefitting from an increased toughness value.

\section{DISCUSSION}

The PEI/PC and PEI/PS model systems described above are meant to demonstrate the feasibility of the rigid-rigid polymer concept. If a quantitative mechanical model is to be established, a more detailed analysis has to be followed. The present work will qualitatively discuss the mechanical conditions for which a rigid polymer can be toughened by another rigid polymer.

The results of the 2-D FEM simulation is based on the modeling of a cylindrical inclusion embedded in a thin plate under uniaxial tension. Under such a condition, when the embedded cylindrical inclusion is a rubber particle or a hole, the stress concentration factor is about three $(4,54)$. In contrast, a stress concentration factor of only about two can be reached for that of a spherical rubber particle or a hole embedded in an infinitely thick plate (55). It is also found that the effects of anelastic and plastic deformations of an inclusion in initiating localized yielding of matrix between a cylindrical inclusion embedded in a thin plate and a spherical inclusion embedded in an infinitely thick plate qualitatively agree with each other $(4,5,43,45)$. Thus, it is 
conceivable that the knowledge learned from the 2-D FEM results is directly applicable to the case where a spherical inclusion is embedded in an infinitely thick plate, if the difference in the induced stress concentration factors between the two conditions is taken into account. Furthermore, since, in reality, the inclusion phase of the multi-phase polymer alloy is approximately spherical, and the size of the inclusion is far smaller than the thickness of the test specimen, it is practical to discuss the present subject using the boundary condition where a spherical inclusion is embedded in an infinitely thick plate. Hereafter, a stress concentration factor of two, instead of three, will be used to describe the effect of yielding and fracture of an inclusion in a continuum matrix.

Based on the present study (plane stress condition) and by assuming that interfacial adhesion and the toughener phase morphology are ideal, three criteria can be established for ensuring a toughened rigid-rigid polymer alloy (the von Mises criterion is assumed):

or

$$
E_{t, i} \leq 1.6 \cdot E_{t, m}
$$

$$
E_{t, m} \leq 1.6 \cdot E_{t, i}
$$

(if the inclusion behaves elastically or anelastically before matrix yielding)

$$
\gamma_{y, i} \cdot S_{\gamma, m} \leq \gamma_{y, m} \cdot S_{\gamma, i}
$$

(if the inclusion tends to shear yield)

$$
\gamma_{b, i} \cdot S_{\gamma, m} \leq \gamma_{y, m} \cdot S_{\gamma, i}
$$

(if the inclusion tends to craze or microcrack) where $E_{i}$ is the tangent modulus; $S_{\gamma}$ is the octahedral shear strain concentration factor; $\gamma_{y}$ and $\gamma_{b}$ denote the octahedral shear yielding strain and brittle strain; the subscripts " $i$ " and " $m$ " denote the inclusion and the matrix, respectively. The significance of the above three criteria is discussed below.

When only the effect of relative tangent moduli of polymer pairs is considered, i.e., criterion (1), Goodier's elastic closed-form solutions (55) can be used. Numerical simulation is not needed. (In this case, the inclusion is spherical and is embedded in an infinitely thick plate.) In order for localized plane strain shear bands to form inside the material, a stress concentration factor of 1.15 has to be reached (38). Hence, based on Goodier's solutions, so long as the tangent modulus (i.e., the slope of its $\sigma-\epsilon$ curve at a given strain state) of the inclusion is ca. $60 \%$ higher or lower than that of the matrix, a sufficient stress concentration will arise and trigger localized shear deformation (see Fig. 1O) in a uniaxial or biaxial tensile stress field. It is noted that in stress states where the hydrostatic mean stress is small, the effect of poisson's ratio differences between the inclusion and the matrix on the stress concentration is found to be minor. Only when the poisson's ratio approaches 0.5 does the stress concentration effect become magnified. Therefore, only tangent moduli
Plot of Tangent Moduli vs. $\tau_{\text {oct }}$ Stress Concentration Factor

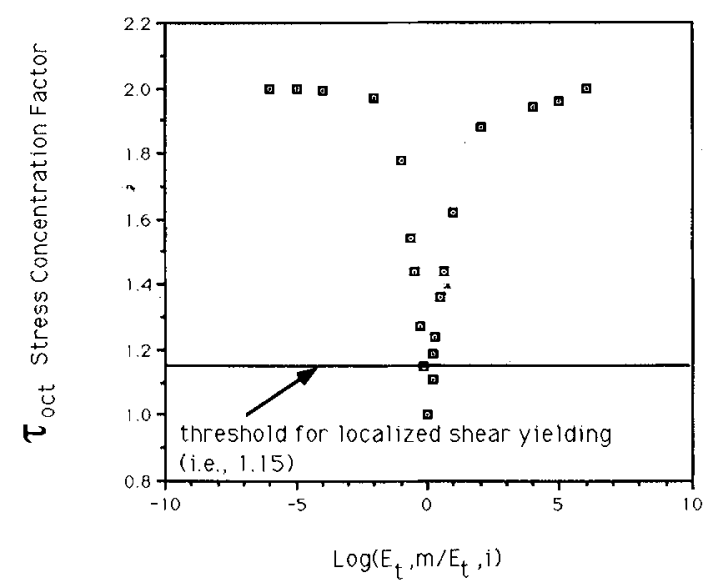

Fig. 10. A plot showing the stress concentration effect due to differences in elastic moduli among the rigid polymer pairs (under the uniaxial loading condition).

are considered here for rigid-rigid polymer toughening in plane stress.

It should be further emphasized that the above criterion is focused on the relative tangent moduli difference, instead of the commonly utilized Young's moduli, between the matrix and inclusion at any given strain level before matrix failure occurs.

Criterion (1) is supported by a recent work on the epoxy/PPO system conducted by Pearson and Yee (56). The stress-strain-dilatation behavior of epoxy, PPO, and epoxy/PPO are shown in Fig. 11. The initial stress concentration factor at low strain level is small, ca. one. Hence, if only the elastic behavior of the polymers is considered, localized shear banding cannot occur, due to the low stress concentration factor. On the other hand, if the tangent moduli of the $\sigma-\epsilon$ curve is considered, at $\approx 0.5 \%$ strain level, the tangent moduli between the two polymers begin to differ. Consequently, the stress concentration at

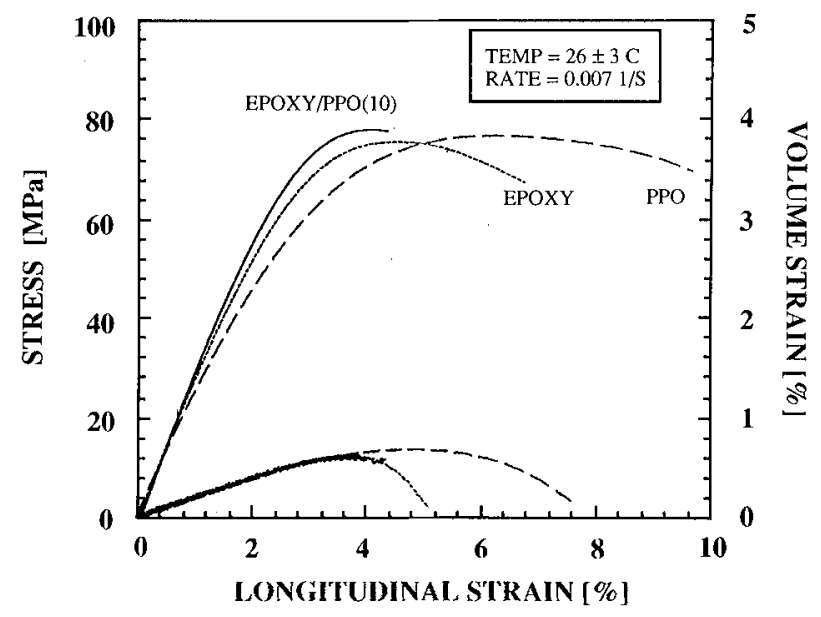

Fig. 11. Stress-strain-dilatation curve of the Epoxy/PPO system. 
the epoxy/PPO interface builds up. When the longitudinal strain approaches about $3.5 \%$, the tangent moduli differ substantially. Localized shear banding takes place (Fig. 12). Collection of these massive localized shear bands in the matrix eventually causes the bulk specimen to form an inclined shear neck. The $\sigma-\epsilon$ curve thus bends over (Fig. 11). In the case of blunt-notch fracture using the double-notch four-point-bend specimen (DN-4PB) (57) of epoxy/PPO system, Pearson and Yee also showed that massive shear banding occurs at the notch tip without any addition of rubber particles (Fig. 13).

The above experiments indicate that in rigid-rigid polymer toughening, when the sample experiences a uniaxial or biaxial tensile stress field, cavitational processes, such as internal cavitation in rubber particles, crazing, debonding, or microcracking, are not needed for triggering localized shear banding. In addition, the elastic Young's modulus seem not to be important. Instead, the tangent modulus at a given strain level before matrix failure occurs appears to be critical for plane stress rigid-rigid polymer toughening.

Nevertheless, if the sample is thick and has a sharp crack in it, when tested under mode-I fracture, the triaxial tension in front of the crack tip has to be relieved in order to activate the shear yielding process, even if the above conditions are met. As shown in Fig. 14, when a sharp crack is introduced in the epoxy/PPO system and tested under DN-4PB condition, only the microcracking type of feature is found at the plane strain region (at the center of the specimen). The above statement also found support from experiments on the PA/PPO system both in the plane stress and plane strain regions (58).

When the conditions described in criterion (1) are not met (e.g., the PEI/PC and PEI/PS systems), then, the condition described by criterion (2) or (3) have to be met for enhancing localized plasticity. Criterion (2) states that if the resultant yield strain (i.e., $\gamma_{y, i}$.

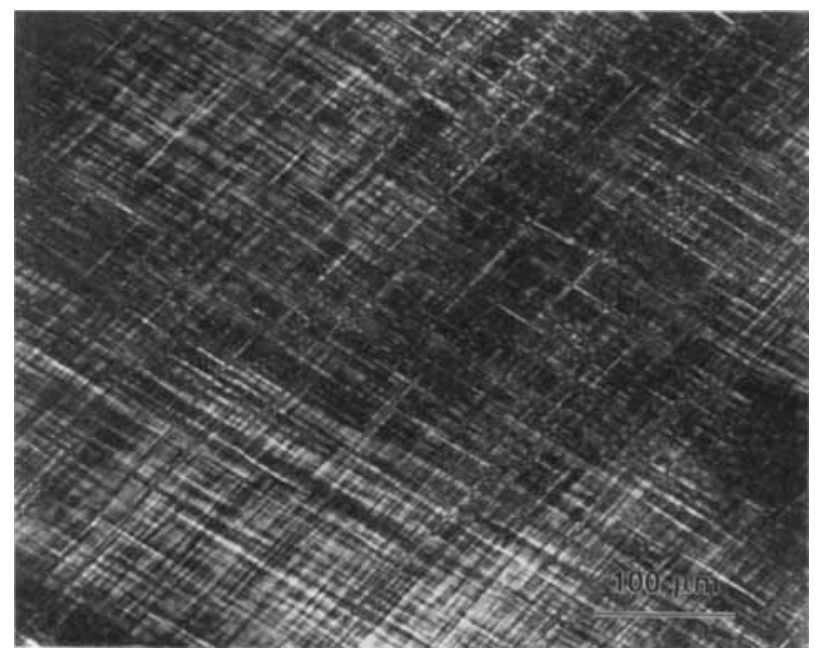

Fig. 12. An optical micrograph taken under cross-polars showing localized shear bands in a tensile specimen of epoxy/PPO.

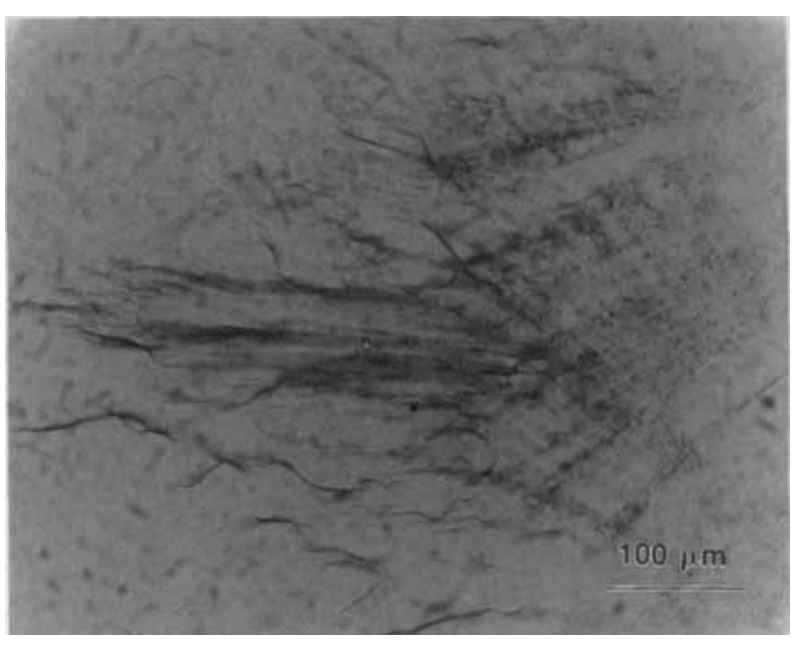

(a)

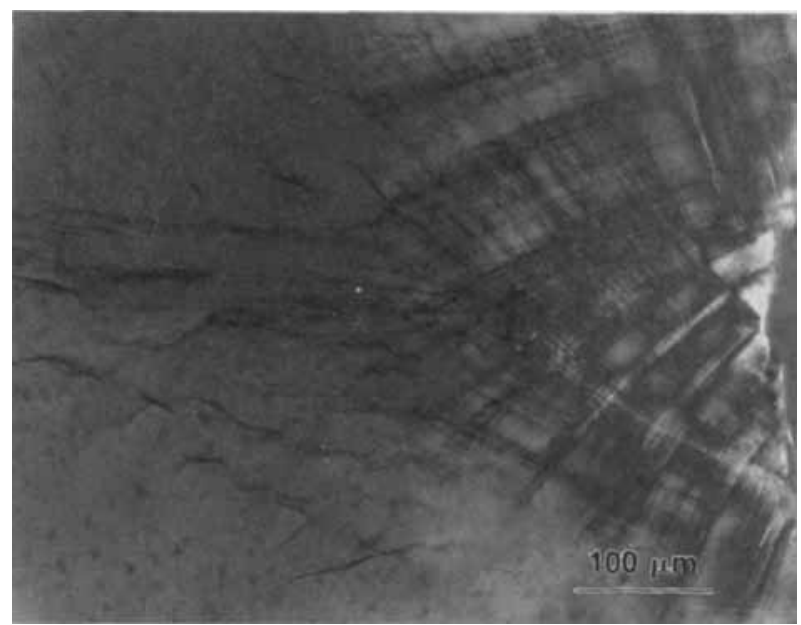

(b)

Fig. 13. Optical micrographs (a) taken under bright field to show signs of microcracking, (b) taken under crosspolars to show the localized shear bands in a blunt-notch $D N-4 P B$ specimen of epoxy/PPO.

$S_{\gamma, m}$ ) of the inclusion is smaller than that of the matrix, then, the inclusion will shear yield first and generate a high stress concentration factor (ca. two) close to that of the rubber particle case at the interface between the matrix and the inclusion. Consequently, localized shear banding at the matrix phase is induced. In other words, the competition between the matrix and the inclusion for an early yielding is governed by not only the yield strains of the two phases, but also the strain amplification due to their counterparts.

Criterion (2) does find support from the work conducted by Sue and Yee on the PA/PPO system (59). The resultant yield strain of rubber-modified PPO is about $18 \%(9 \% \cdot 2=18 \%)$, while that of the moisture equilibrated PA is $\geq 25 \%$. As a result, the PPO undergoes shear yielding first, which causes the stress concentration to build up abruptly, followed 


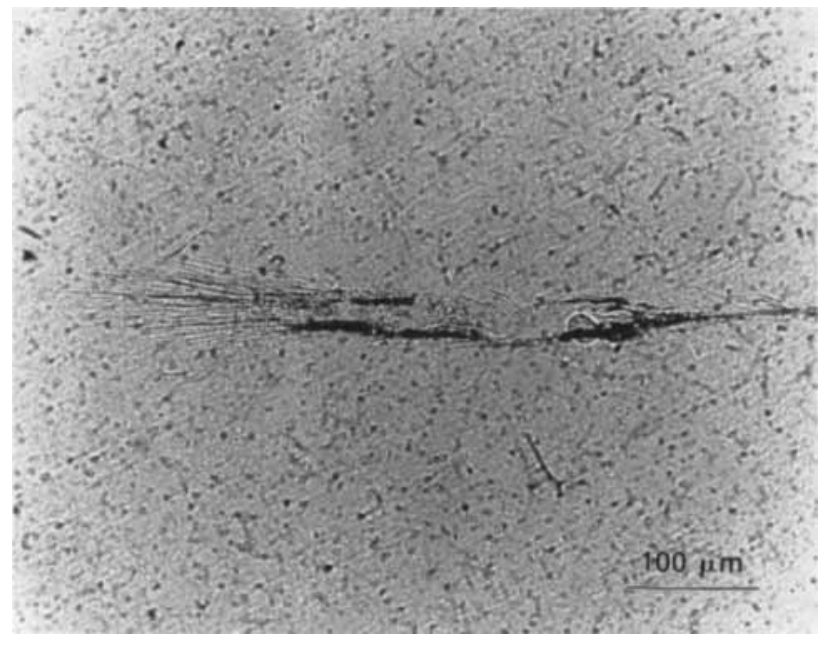

Fig. 14. An optical micrograph taken under bright field showing the presence of microcracks at a sharp-crack tip (DN-4PB specimen of epoxy/PPO).

by the shear yielding of the PA matrix. The PA/PPO alloy is thus toughened.

For criterion (3), the postulate we proposed has not been verified. However, it is intuitive that if crazing or microcracking of the inclusion takes place earlier than the failure of the matrix, potentially the stress concentration build-up should be similar to that of the 2-D FEM prediction. It is also intuitive to the authors that if debonding at the matrix and inclusion occurs at an appropriate moment, the stress concentration factor can also rise to about two (4). As a result, toughening of the rigid-rigid polymer alloy system can be achieved. Again, it is important to re-emphasize that the above discussions only hold for uniaxial and biaxial tensile stress conditions.

The criteria for the selection of toughened rigidrigid polymer alloys obtained in this study also find support from other experimental investigations $(8$, 9). In cases where the specimens are tested under plane stress conditions, i.e., either uniaxially and biaxially, the 2-D FEM simulation results need not be modified. The experimental works on PC/PSAN (9) and PC/PMMA (8), which support the criteria proposed in this study, have found these alloys to be tougher than PC.

For the plane strain condition, e.g., in front of the crack tip, even if the polymer pairs meet the criteria suggested in this study, the triaxial tension still has to be relieved prior to the formation of localized shear yielding. Experimental work (59) conducted on $\mathrm{PA} / \mathrm{PPO}^{*}$ supports this point. The elastomeric phase inside the PPO particles initiates numerous crazes in front of the crack tip, when the specimen is loaded. These crazes propagate from the PPO particles into the PA matrix. The triaxial tension in front of the crack tip is therefore relieved due to the

*PA has a higher yield strain than the yield (craze) strain of rubber-modified $\mathrm{PPO}$. formation of these crazes. As a result, the PPO particles undergo shear yielding and cause the surrounding PA matrix to shear yield. The PA/PPO alloy is thus toughened.

The 2-D FEM results on the PEI/PC and PEI/PS systems are likely to be valid. However, the 2-D FEM simulation work does not take the triaxial tension effect into account. Based on the rigid-rigid polymer toughening concept mentioned earlier, to trigger shear yielding in either inclusion or matrix polymers, the triaxial tension has to be relieved. Therefore, localized shear yielding in PEI/PC and PEI/PS systems may not occur until the relief of the triaxial tension happens. If the craze formation of the PS inclusion is sufficient to relieve the triaxial tension, the 2-D FEM results on the PEI/PS system may still hold.

In the case of the PEI/PC system, if the triaxial tension is not relieved, the PC inclusion cannot yield under the constrained condition. Nevertheless, upon debonding at the interface or cavitation of a thirdphase elastomer taking place inside the PC inclusion, the PC inclusion can still yield to cause an abrupt increase in stress concentration around the PEI matrix, and thus, widespread shear banding may occur and toughen the matrix.

The present work is meant to gain some preliminary understanding and insight for the selection of rigid-rigid polymer pairs. The simulation procedures conducted in this study involve many rather simplified assumptions. The outcome of the 2-D FEM results should therefore be treated with some caution. Nevertheless, the simulation results appear to be physically sound and have gained support from various experimental investigations. As a result, the criteria for the selection of toughened rigid-rigid polymer alloys are likely to be qualitatively correct for plane stress conditions.

\section{CONCLUSION}

Two-dimensional FEM simulations, which involve consideration of the nonlinear behavior of a material, have been conducted on some two-phase polymer alloy systems. The results suggest that if the rigid toughener phase polymers possess either 1) $60 \%$ higher or lower elastic modulus (under uniaxial tension) compared with the matrix, or 2) smaller yield (or crazing) strain than, but comparable yielding stress to, the matrix, then a sufficient stress concentration around the toughener phase will arise and cause the matrix to undergo localized shear deformation. However, the above statements will not be valid if the specimen is under the plane strain condition. In this case, the triaxial tension still has to be relieved prior to the formation of localized shear bands. The current study further supports the rigid-rigid polymer toughening concept we proposed.

\section{ACKNOWLEDGMENTS}

This work is partially supported by the National Science Foundation (Grant No. DMR-8708405), the 
E.I. Du Pont de Nemours \& Company, and the Macromolecular Research Center of the University of Michigan.

\section{REFERENCES}

1. A. F. Yee, D. S. Parker, H. J. Sue, and I-C. Huang, "Toughening Mechanisms in Some Rubber/Plastic and Plastic/Plastic Multi-phase Blends," PMSE Div., Preprints, 194th ACS National Meeting, Aug. 1987.

2. H. J. Sue and A. F. Yee, "Toughening Mechanism\{s in Alloys of Rigid Polymers," SPERETEC (Chicago), The Society of Plastics Engineers, Sept. 1987.

3. H. J. Sue and A. F. Yee, "Toughening Mechanisms in Alloys of Rigid Polymers," 7th International Conference on Deformation. Yield and Fracture of Polymers, Cambridge, England, April 1988.

4. H. J. Sue, PhD thesis, The University of Michigan, Ann Arbor, Mich. (1988).

5. R. A. Pearson, PhD thesis, The University of Michigan, Ann Arbor, Mich. (1990).

6. S. Y. Hobbs and M. E. J. Dekkers, J. Mater. Sci., 24, 1316 (1989).

7. M. E. J. Dekkers, S. Y. Hobbs, V. H. Watkins, J. Mater. Sci., 23, 1225 (1988).

8. T. Kurauchi and T. Ohta, J. Mater. Sci., 19, 1699 (1984).

9. K. Koo, T. Inoue, and K. Miyasaka, Polym. Eng. Sci., 25, 741 (1985).

10. A. S. Wood, Modern Plastics, April 1985, p. 81.

11. C. B. Bucknall and A. H. Gilbert, Polymer, 30, 213 (1989).

12. A. Ghaffar, C. Sadrmohaghegh, and G. Scott, Europ. Polym. J., 17, 941 (1981).

13. C. Sadrmohaghegh, G. Scott, and E. Seroudeh, Europ. Polym. J., 19, 19 (1983).

14. M. E. J. Dekkers and S. Y. Hobbs, Polym. Eng. Sci., 27, 1164 (1987)

15. Y. Fujita, K. Koo, J. Angola, T. Inoue, and T. Sakai, Kobunshi Ronbunshu, 43, 119 (1987).

16. H. D. Stenzenberger, W. Romer, P. M. Hergenrother, and B. J. Jensen, SAMPE Conf., Covina, Calif. 34 2054 (1989).

17. T. Ishikawa, Netsu Kokasei Jushi, 10, 117 (1989).

18. Y. Sun and Z. Fu, Am. Chem. Soc., Div. Polym. Chem. Polym. Prep., 29, 177 (1988).

19. N. Shibuya, Y. Sobajima, and H. Sano, European Patent Application 0225170 (1987).

20. a) A. F. Yee and R. A. Pearson, J. Mater. Sci., 21, 2462 (1986).

b) R. A. Pearson, A. F. Yee, J. Mater. Sci., 21, 2475 (1986).

21. G. T. Hahn and A. R. Rosenfield, Metall. Trans. A, 6A, 653 (1975).

22. S. H. Goods and L. M. Brown, Acta Metall., 27, 1 (1979).

23. A. S. Argon, J. Im, and A. Needleman, Metall. Trans. A, 6A, 815 (1975)

24. A. S. Argon, J. Im, and R. Safoglu, Metall. Trans. A, 6A, $825(1975)$

25. A. S. Argon, J. Im, Metall. Trans, A, 6A, 839 (1975).
26. R. W. Coade, J. R. Griffiths, and B. A. Parker, Philo. Mag., 44, 357 (1981).

27. L. J. Walpole, J. Mech. Phys. Solids, 18, 343 (1970).

28. M. Shibata and K. Ono, Acta Metall., 26, 921 (1978).

29. B. Karlsson, and B. O. Sundstrom, Mater. Sci. Eng., 16, 161 (1974).

30. K. Tanaka and T. Mori. Acta Metall., 18, 931 (1978).

31. K. Tanaka, K. Narita, and T. Mori, Acta Metall., 20 , 297 (1978).

32. A. G. Evans and R. M. Cannon, Acta Metall., 34, 761 (1986).

33. R. M. McMeeking and A. G. Evans, J. Am. Cera. Soc., 65, 242 (1982).

34. A. G. Evans and K. T. Faber, J. Am. Cera. Soc., 67, 255 (1984).

35. I. W. Chen and P. E. Reyes-Morel, J. Am. Cera. Soc., 69, 181 (1986).

36. R. J. Oxborough and P. B. Bowden, Philo. Mag., 28, 547 (1973).

37. A. S. Argon and M. I. Bessonov, Philo. Mag., 35, 917 (1977).

38. P. B. Bowden, in The Physics of Glassy Polymers, ch. 5, R. Haward ed., Appl. Sci. Pub., London (1973).

39. T. Brady and G. Yeh, J. Macromol. Sci. (Phys.), B9, 659 (1974).

40. R. E. Robertson, Appl. Polym. Symp. 7, 201 (1968).

41. R. E. Robertson and A. M. Patel, Polym. Eng. Sci., 12 , 345 (1972)

42. T. T. Wang, M. Matsuo, and T. K. Kwei, J. Appl. Phys., 42, 4188 (1971)

43. C. B. Bucknall, D. Clayton, and W. E. Keast, J. Mater. Sci., 7. 1443 (1972).

44. M. Matsuo, T. T. Wang, T. K. Kwei, J. Polym. Sci., A-2, 10, 1085 (1972)

45. M. E. J. Dekkers, PhD thesis, Eindhoven Univ. of Tech., Germany (1985).

46. L. J. Broutman and G. Panizza, Inter. J. Polym. Mater., 1, 95 (1971).

47. L. Bevan, J. Polym. Sci., Polym. Phys. Ed., 19, 1759 (1981).

48. B. D. Agarwal and L. J. Broutman, Fiber Sci. Technol., 7, 63 (1974).

49. T. Ricco, A. Pavan, and F. Danusso, Polym. Eng. Sci., 18, $774(1978)$.

50. O. C. Zienkiewicz, The Finite Element Method, McGraw-Hill, New York (1977).

51. H. J. Sue and A. F. Yee, Polymer, 29, 1619 (1988).

52. S. S. Sternstein, in Treatise on Materials Science and Technology, vol. 10, part IV, J. M. Schultz, ed., Academic Press, New York (1977).

53. R. P. Nimmer, Polym. Eng. Sci., 27, 16 (1987).

54. W. A. Nash, Strength of Materials, 2nd ed., p. 385, McGraw-Hill, New York (1972).

55. J. N. Goodier, J. Appl. Mech., 1, 39 (1933).

56. R. A. Pearson and A. F. Yee, to be published.

57. H. J. Sue, R. A. Pearson, D. S. Parker, J. Huang, and A. F. Yee, Am. Chem. Soc. Div. Polym. Chem. Polym. Prep. 28, 147 (1988).

58. H. J. Sue and A. F. Yee, accepted by J. Mater, Sci., July 1990.

59. H. J. Sue and A. F. Yee, J. Mater. Sci., 24, 1447 (1989). 